\title{
Breastfeeding duration and reduced risk of NAFLD in midlife of parous women
}

\author{
Alessandro Mantovani $^{{ }^{*}}$ (]) Giorgia Beatrice ${ }^{1}$, Chiara Zusi ${ }^{1,2}$, Andrea Dalbeni ${ }^{3}[$ \\ ${ }^{1}$ Section of Endocrinology, Diabetes and Metabolism, Department of Medicine, University and Azienda Ospedaliera Universitaria \\ Integrata of Verona, 37126 Verona, Italy \\ ${ }^{2}$ Pediatric Diabetes and Metabolic Disorders Unit, Department of Surgical Sciences, Dentistry, and Pediatrics, and Gynaecology, \\ University Hospital of Verona, 37126 Verona, Italy \\ ${ }^{3}$ Section of General Medicine, Hypertension and Liver Unit, University and Azienda Ospedaliera Universitaria Integrata of \\ Verona, 37126 Verona, Italy
}

*Correspondence: Alessandro Mantovani, Section of Endocrinology, Diabetes and Metabolism, University and Azienda Ospedaliera Universitaria Integrata Piazzale A. Stefani, 37126 Verona, Italy. alessandro.mantovani@univr.it

Academic Editor: Lindsay A. Farrer, Boston University School of Medicine, USA

Received: August 20, 2021 Accepted: August 26, 2021 Published: October 31, 2021

Cite this article: Mantovani A, Beatrice G, Zusi C, Dalbeni A. Breastfeeding duration and reduced risk of NAFLD in midlife of parous women. Explor Med. 2021;2:378-81. https://doi.org/10.37349/emed.2021.00056

\section{Keywords}

Breastfeeding, nonalcoholic fatty liver disease, metabolic associated fatty liver disease

Nonalcoholic fatty liver disease (NAFLD) has currently become a relevant public health challenge, since it is the most chronic liver disease observed in clinical practice worldwide and is associated with serious hepatic (such as liver failure and hepatocellular carcinoma) and extra-hepatic complications, including cardiovascular diseases and type 2 diabetes mellitus (T2DM) [1]. The pathophysiology of NAFLD is complex, as several endogenous (e.g., genetic variants, age, sex, ethnicity and gut microbiota) and exogenous (e.g., metabolic features, lifestyle habits, alcohol, and fructose) factors play a role in the development and progression of NAFLD and its advanced forms [1]. Recently, it has been also suggested that a short duration of breastfeeding (ideally less than 6 months) might be independently associated with an increased risk of NAFLD in later life of parous women. Hypothetically, this may be due to the fact that a short duration of breastfeeding is associated with fat accumulation, weight gain and higher risk of some cardio-metabolic disorders [2, 3].

To our knowledge, at present, only two cross-sectional studies have explored the association between breastfeeding duration and risk of NAFLD in later life of parous women (Table S1). In a 2019 cross-sectional study enrolling 844 US women [median age 25 years; median body mass index (BMI) $22 \mathrm{~kg} / \mathrm{m}^{2}$ ] from the Coronary Artery Risk Development in Young Adults cohort study, who delivered $\geq 1$ child and underwent computed tomography quantification of hepatic steatosis after 25 years, Ajmera et al. [4] showed that a breastfeeding duration longer than 6 months, compared to a breastfeeding duration less than 1 month, was associated with a lower risk of NAFLD in later life of parous women, independent of BMI, ethnicity, parity and lifestyle habits [adjusted odds ratio $0.46,95 \%$ confidence interval (CI) 0.22-0.97]. More recently, in a cross-sectional study involving 6,893 Korean parous women aged 30-50 years from the Korean National Health and Nutrition Examination Survey, Park et al. [5] reported that longer breastfeeding was associated with a protective effect against NAFLD (as detected by hepatic steatosis index) in later life of parous women,

(C) The Author(s) 2021. This is an Open Access article licensed under a Creative Commons Attribution 4.0 International License (https://creativecommons.org/licenses/by/4.0/), which permits unrestricted use, sharing, adaptation, distribution and reproduction in any medium or format, for any purpose, even commercially, as long as you give appropriate credit to the original author(s) and the source, provide a link to the Creative Commons license, and indicate if changes were made. 
independent of age, BMI, waist circumference, dyslipidemia, T2DM, hypertension, education levels, smoking status, age at menarche, age at the first delivery and age at the last delivery [adjusted odds ratio 0.67 (95\% CI 0.48-0.94) for women with $\geq 6$ to $<12$ months of breastfeeding duration and adjusted odds ratio 0.64 (95\% CI 0.46-0.89) for women with $\geq 12$ months of breastfeeding duration, when compared to women with $<1$ month of breastfeeding duration].

With the aim to assess the magnitude of the effect of breastfeeding duration on the risk of NAFLD (as diagnosed by imaging techniques or indirect biomarkers) in later life of parous women, we have meta-analyzed the data of the aforementioned studies [4,5]. More specifically, for both studies, we have obtained the adjusted odds ratios for the effect of breastfeeding duration longer than 6 months on the risk of NAFLD in later life of parous women, when compared to the breastfeeding that lasted less than 1 month. These odds ratios were pooled and an overall estimate of effect size was computed using the random-effects restricted maximum likelihood (REML) model. All statistical tests were two sided and used a significance level of $P<0.05$. We used STATA® software v16.1 (StataCorp, College Station, Texas, USA) for statistical analyses.

As reported in Figure 1, compared to the breastfeeding that lasted less than 1 month, breastfeeding longer than 6 months was independently associated with a 37\% lower risk of NAFLD in later life of parous women (random-effects odds ratio $0.63,95 \%$ CI $0.51-0.79, I^{2}=0 \%$ ). Despite the limitations of this analysis (including the number of the eligible studies and the choice to take the adjusted odds ratio of model 8 in the study by Ajmera et al. [4]), this result corroborates the maternal benefit of breastfeeding longer than 6 months against NAFLD and provides the magnitude of the effect.

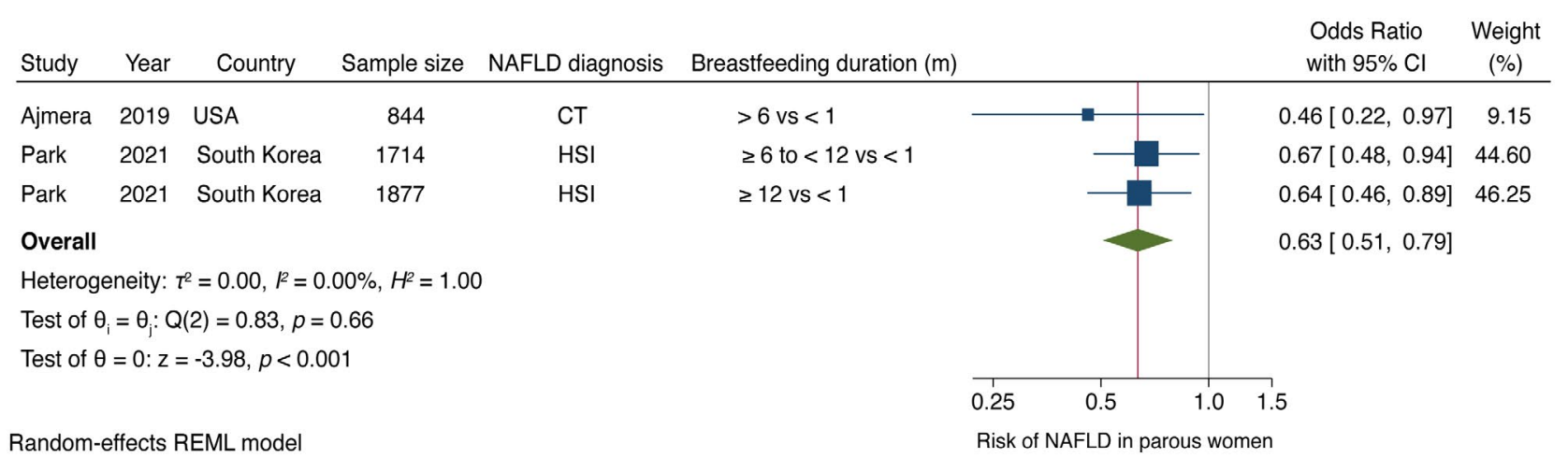

Figure 1. Forest plots and pool ed estimates of the effects of breastfeeding duration longer than 6 months on the risk of NAFLD (as detected by imaging techniques or indirect biomarkers) in later life of parous women, when compared to the breastfeeding that lasted less than 1 month ( $n=2$ studies). Note: regarding the study by Ajmera et al. [4], we have chosen the adjusted odds ratio reported in the model 8 . The result, however, remained substantially similar when the odds ratios of the other adjusted models were taken. CT: computed tomography; HSI: hepatic steatosis index

It is known that breastfeeding, especially when it lasts more than 6 months, helps to promote healthy maternal metabolism [6]. However, to date, the biological mechanisms underpinning the association between breastfeeding duration and reduced risk of NAFLD in parous women remain uncertain. That said, as also discussed elsewhere [4-7], some epidemiological studies currently indicate that women who breastfeed have a reduced risk of some chronic disease [6], such as T2DM, hypertension and metabolic syndrome; all conditions strongly and frequently associated with NAFLD and its advanced forms [1]. Moreover, experimental studies have also reported that breastfeeding may improve insulin sensitivity and mobilize fat stores, thereby reducing the risk of NAFLD [6].

Collectively, the observational studies available so far and the result of our analysis reinforce the assertion that breastfeeding is relevant for the long-term health of the mother, with a protective effect on the risk of NAFLD. However, despite this evidence, along with other Authors [4-7], we believe that future observational studies on this issue are timely needed and that other aspects should be considered, such as the complexity of the pathogenesis of NAFLD (evaluating also the genetic variants linked to hepatic steatosis), intensity of breastfeeding, various socioeconomic and cultural factors, pre-conception background and pre-natal care for 
the duration of pregnancy. A qualitative leap in understanding the role of breastfeeding and its relationship with the risk of NAFLD should require a multidisciplinary approach across different medical disciplines.

\section{Abbreviations}

BMI: body mass index

CI: confidence interval

NAFLD: nonalcoholic fatty liver disease

T2DM: type 2 diabetes mellitus

\section{Supplementary materials}

The supplementary material for this article is available at: https://www.explorationpub.com/uploads/ Article/file/100156_sup_1.pdf.

\section{Declarations}

\section{Author contributions}

AM conceived and designed the study. AM, GB, CZ, and AD researched data and reviewed/edited the manuscript. AM analyzed the data and wrote the manuscript draft. All Authors approved the final version of the manuscript.

\section{Conflicts of interest}

The authors have no potential conflicts of interest to disclose.

Ethical approval

Not applicable.

\section{Consent to participate}

Not applicable.

\section{Consent to publication}

Not applicable.

Availability of data and materials

Not applicable.

\section{Funding}

Not applicable.

\section{Copyright}

(c) The Author(s) 2021.

\section{References}

1. Targher G, Tilg H, Byrne CD. Non-alcoholic fatty liver disease: a multisystem disease requiring a multidisciplinary and holistic approach. Lancet Gastroenterol Hepatol. 2021;6:578-88.

2. Lima NP, Bassani DG, Magalhaes EIS, Barros FC, Horta BL. Breastfeeding and maternal cardiovascular risk factors: 1982 Pelotas Birth Cohort. Sci Rep. 2019;9:13092.

3. Suliga E, Ciesla E, Gluszek-Osuch M, Lysek-Gladysinska M, Wawrzycka I, Gluszek S. Breastfeeding and prevalence of metabolic syndrome among perimenopausal women. Nutrients. 2020;12:2691. 
4. Ajmera VH, Terrault NA, VanWagner LB, Sarkar M, Lewis CE, Carr JJ, et al. Longer lactation duration is associated with decreased prevalence of non-alcoholic fatty liver disease in women. J Hepatol. 2019;70:126-32.

5. Park Y, Sinn DH, Oh JH, Goh MJ, Kim K, Kang W, et al. The association between breastfeeding and nonalcoholic fatty liver disease in parous women: a nationwide cohort study. Hepatology. 2021; [Epub ahead of print].

6. Nobili V, Schwimmer JB, Vajro P. Breastfeeding and NAFLD from the maternal side of the mother-infant dyad. J Hepatol. 2019;70:13-4.

7. Hershman M, Mei R, Kushner T. Implications of nonalcoholic fatty liver disease on pregnancy and maternal and child outcomes. Gastroenterol Hepatol (N Y). 2019;15:221-8. 